\title{
Costaricanischer Impuls für Deutschland: der „Femizid“ als eigener Straftatbestand
}

\author{
Sabine Kräuter-Stockton \\ Mitglied der Kommission Strafrecht, Leiterin der djb-Fachgruppe \\ Straftaten gegen Ältere, Staatsanwältin/Gruppenleiterin, Saar- \\ brücken
}

Am 9. und 10. August 2012 organisierten die deutsche und die französische Botschaft in Costa Rica ein zweitägiges Forum zum Thema „Bekämpfung und Prävention von Gewalt gegen Frauen“ in der Hauptstadt San José. Als Referentinnen aus Europa waren die Französin Michèle Vianès, Präsidentin der Nichtregierungsorganisation „Regards de femmes“, und Sabine Kräuter-Stockton, Staatsanwältin in Saarbrücken und Mitglied der Kommission Strafrecht des djb, eingeladen. Die zweitägige Veranstaltung war gut besucht: In dem kleinen Land in Mittelamerika, das gerade einmal knapp viereinhalb Millionen Einwohner/innen zählt, leben zahlreiche kluge, feministisch orientierte und wortgewandte Wissenschaftlerinnen, Praktikerinnen und Politikerinnen, die sich teils vom Podium aus, teils aus dem Publikum engagiert beteiligten.

Gewalt ist in Costa Rica, das sich an der Durchgangsstrecke zwischen dem Drogenlieferanten Kolumbien und dem Abnehmerland USA befindet und in den letzten Jahren zunehmend von Drogenkriminalität heimgesucht wird, sicherlich weiter verbreitet als in Europa. Zudem ist hier, wie überall in Lateinamerika, die Kultur stark an der patriarchalen Tradition ausgerichtet. Doch auf der anderen Seite ist San José der Sitz des wichtigen Interamerikanischen Gerichtshofs für Menschenrechte, der für aufsehenerregende Urteile in Sachen Frauenrechte zeichnet und der an der Menschenrechtsthematik interessierte Menschen in die Stadt zieht. Hinzu kommt, dass Costa Rica Unterzeichnerstaat der Interamerikanischen „Konvention zur Verhinderung, Bestrafung und Eliminierung von Gewalt gegen Frauen“ ist. Das Land hat im Übrigen 1949 seine Armee komplett abgeschafft und wird heute von Staatspräsidentin Laura Chinchilla regiert.

Vergleichbare Widersprüchlichkeiten lassen sich in Costa Rica auch bei der Bekämpfung der Gewalt gegen Frauen ausmachen: Während die praktische Umsetzung - so wurde von allen Seiten beklagt - an mangelnder Effizienz, teilweise auch am fehlenden Willen der Akteure zu einer echten Änderung krankt, ist die Gesetzeslage, was die Bekämpfung von Gewalt gegen Frauen angeht, schon seit Jahrzehnten fortschrittlich und kann als Vorbild oder Inspiration für europäische Gesetzgeber dienen.

Schon 1996, als bei uns das Gewaltschutzgesetz noch in weiter Ferne lag, trat in Costa Rica das „Gesetz gegen häusliche Gewalt“ (Ley contra la violencia doméstica ${ }^{1}$ ) in Kraft, ein Gesetz, das unter den Begriff „häusliche Gewalt“ auch psychische und sexuelle Gewalt subsumierte, das schon damals

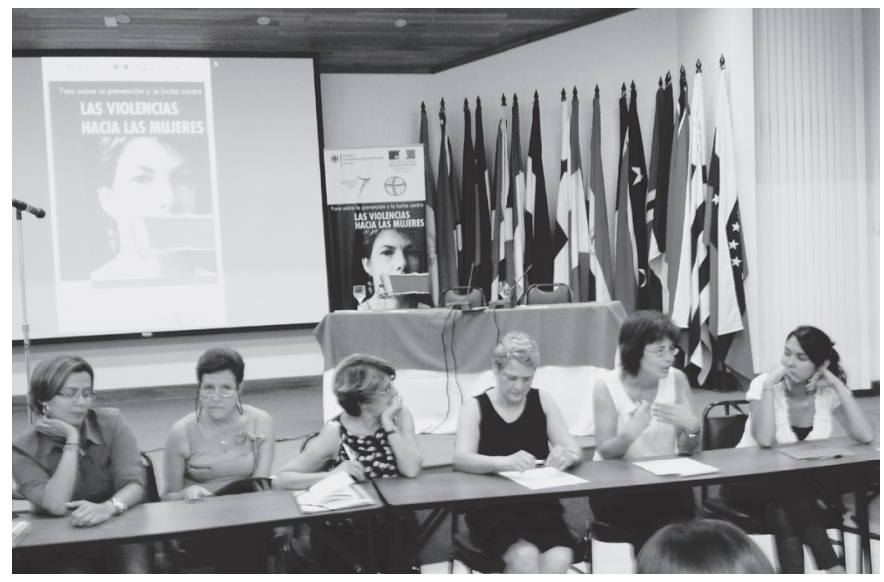

$\Delta$ Das Forum fand im „Salón de vidrio“ (Glassaal) des Interamerikanischen Instituts für Menschenrechte statt. Sabine Kräuter-Stockton (zweite von rechts) referiert. Dritte von links: Michèle Vianès, Präsidentin Regards de Femmes, Lyon/Frankreich.

Schutzmaßnahmen wie eine Kontaktsperre oder die polizeiliche und gerichtliche Wegweisung des Täters für sechs Monate aus der gemeinsamen Wohnung vorsah, mit dem darüber hinaus die vorläufige Entziehung des Sorge- oder Umgangsrechts des Täters betreffend die gemeinsamen Kinder sowie Beschlagnahmemöglichkeiten in sein Vermögen zur Sicherung der Unterhaltsansprüche des Opfers und der Kinder geregelt wurden.

Im Mai 2007 trat in Costa Rica das Gesetz über die „Strafbarkeit von Gewalt gegen Frauen“ (Ley de Penalización de la Violencia contra las Mujeres ${ }^{2}$ ) in Kraft, welches sich in der Benennung seiner Ziele auf die CEDAW-Konvention sowie auf die interamerikanische „Konvention zur Verhinderung, Bestrafung und Eliminierung von Gewalt gegen Frauen "3 stützt. Das Gesetz schreibt in seinem Artikel 4 vor, dass Gewaltstraftaten gegen Frauen stets von Amts wegen zu verfolgen und bestimmte Gesichtspunkte als strafverschärfend zu berücksichtigen sind ${ }^{4}$ : Wenn es sich bei den Opfern um behinderte Frauen oder um Frauen handelt, die älter als 65 Jahre sind, oder um schwangere Frauen, oder - ein sehr moderner Gedanke - wenn der gewalttätige Übergriff in Anwesenheit von Kindern des Opfers oder des Täters erfolgt ist.

Weiter wurde in Artikel 21 des Gesetzes ein eigener Straftatbestand des „Femizids“ geschaffen: Mit Freiheitsstra-

1 <http://www.hsph.harvard.edu/population/domesticviolence/costarica.dv.96.pdf $\rangle$ (Zugriff: 17.10.2012).

2 <http://ministeriopublico.poder-judicial.go.cr/coop-intern/Normativa\%20Nacional/o4-Delitos\%20Sexuales/o4.pdf > (Zugriff: 17.10.2012).

3 〈http://www.summit-americas.org/Belemdopara.htm> (Zugriff: 17.10.2012).

4 Erhöhung der Strafe um bis zu einem Drittel: Ley de Penalización de la Violencia contra las Mujeres, Art. 8. 
fe zwischen 20 und 35 Jahren wird derjenige belegt, der eine Frau tötet, mit der er verheiratet ist oder als Lebensgefährte zusammen lebt. ${ }^{5}$

Wozu ein eigener Straftatbestand für die Tötung einer Frau? - mag man da als Ausländerin fragen. Das Töten eines Menschen, also Mord oder Totschlag, ist doch ohnehin in allen Gesetzbüchern schon mit den höchsten Strafen belegt! Wozu ist da noch eine Strafschärfung nötig? Die Costaricanerinnen antworten hier mit einer Gegenfrage: Kennen wir in Deutschland nicht auch die Fälle, in denen der Täter seine Ehefrau und vielleicht auch noch die Kinder umbringt, und dann aber vor Gericht unter Hinweis auf die überaus emotionale psychische Ausnahmesituation, in der er sich unmittelbar vor und während der Tatbegehung befunden hatte (hat doch das Opfer mit Trennung gedroht!) eine starke Strafmilderung erhält?! Dem würde der Gedanke des Femizids eine Strafschärfung entgegensetzen, so dass am Ende doch eine angemessenere Strafe stehen könnte.

Den feministisch engagierten Costaricanerinnen geht aber die Gesetzesfassung der „Ley de penalización“ nicht weit genug. Zunächst wird kritisiert, dass das Gesetz nur die Tötung von (Ehe-)Partnern erfasst und nicht auch Taten in beendeten Beziehungen. Wichtiger scheint aber, dass bei der Gesetzesfassung nicht zum Ausdruck kommt, dass ein Femizid die besonderen Umstände der Tötung der Frau beschreibt, dass die Frau gerade deswegen getötet wurde, weil sie eine Frau ist. Es handelt sich bei dem Femizid um einen Ausdruck der ungleichen Macht- und Herrschaftsverhältnisse zwischen den Geschlechtern und die maximale Ausprägung der häuslichen Gewalt. Besonders wird kritisiert, dass diese nur mittelmäßige
Gesetzgebung dann in der Praxis noch nicht einmal mit dem angemessenen Nachdruck umgesetzt werde.

Hier schließt sich der Kreis: Jedes Gesetz ist nur so gut oder schlecht, wie es angewendet wird. Angesichts der Zahlen in Deutschland würde es uns gut anstehen, den Impuls aus Mittelamerika aufzunehmen und eine Diskussion in Richtung der Schaffung eines eigenen Straftatbestands Femizid zu führen: Im Jahr 2011 wurden in Deutschland 154 Frauen von ihrem Mann, Lebensgefährten oder Ex-Partner umgebracht ${ }^{6}$, was uns zu einer Reaktion drängen muss. Von der Einführung des neuen Straftatbestands wäre nicht nur zu erwarten, dass sie voraussichtlich die Chance einer angemessenen Bestrafung der Täter erhöhen und damit general- und spezialpräventiv wirken würde. Hinzu kommt der nicht zu unterschätzende Effekt, den die Diskussion in der (Fach-)Öffentlichkeit um die Schaffung eines neuen Gesetzes zwangsläufig mit sich bringt, indem die hinter dem diskutierten Gesetzentwurf stehende Realität für die Gesellschaft offengelegt und verdeutlicht wird. Gleichzeitig muss aber unbedingt die Aus- und Fortbildung der Rechtsanwender/innen zu der Thematik „Gewalt gegen Frauen“ verstärkt werden, um der Praxis mehr Rechtssicherheit zu geben und um zu erreichen, dass sie - anders als in Costa Rica - die Intentionen und Möglichkeiten des Gesetzes ausschöpft und nicht an ihnen vorbei handelt.

5 Zum Vergleich: In Art. 111 des costaricanischen Strafgesetzbuches ist Totschlag mit zwischen 12 und 18 Jahren Freiheitsstrafe bewehrt, Art. 112 sieht für verschärfte Fälle (teilweise vergleichbar mit dem deutschen „Mord“) Freiheitsstrafen zwischen 20 und 35 Jahren vor.

6 Zitiert nach Süddeutsche Zeitung vom 23.5.2012, <http://www.sueddeutsche.de/panorama/frauenmorde-sind-haeufig-beziehungstaten-toedliche-zweisamkeit-1.1365223> (Zugriff: 17.10.2012).

\section{Deutscher Juristentag in München, 17. bis 21. September 2012}

\section{Anke Gimbal \\ Geschäftsführerin des djb, Berlin}

Vom 17. bis 21. September 2012 tagte der 69. Deutsche Juristentag (djt) mit ca. 2.000 Teilnehmerinnen und Teilnehmern in München. Kurz vor Beginn des Oktoberfestes waren die Hotelpreise - in München ohnehin hoch - astronomisch, was sicher die oder den ein/e/n oder andere/n von der Teilnahme abgehalten hat. Der „Oktoberfestabend“, zu dem der Juristentag zusammen mit dem Bundesministerium der Justiz für den Dienstag im Münchner Hofbräuhaus eingeladen hatte, fand jedoch großen Zuspruch. Anstelle des schwarzen Anzugs trug man Dirndl oder Lederhose. Statt Prosecco und Sushi, Tomate-Mozzarella oder Ähnlichem gab es Maßkrüge mit hauseigenem Bier, Brotzeitbrettl und ein Haxn-Buffet.

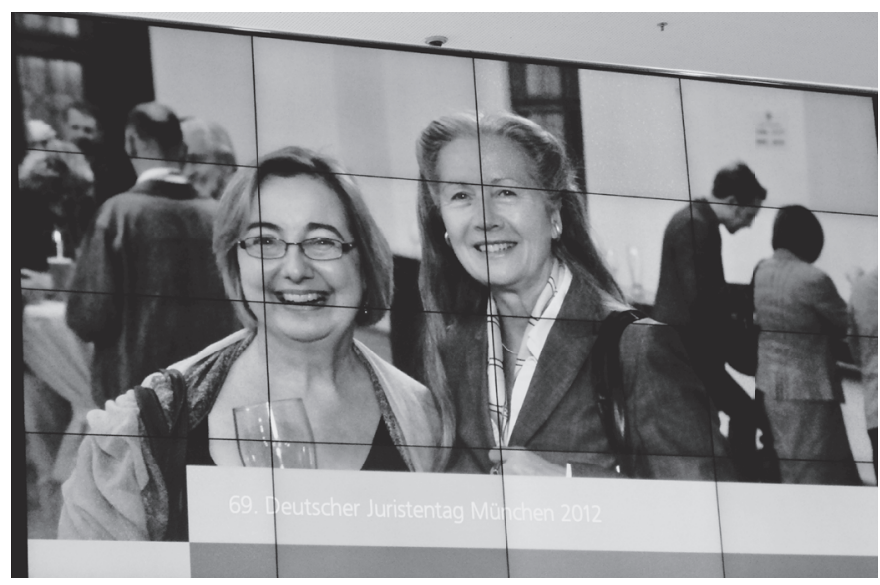

- Präsidentin Ramona Pisal (rechts) mit Rechtsanwältin Petra Heinicke, 1. Vorsitzende des Münchener Anwaltvereins, djb-Bundesvorstandsmitglied von 1993 bis 1995. (Foto: CD, djb) 